\title{
TOPOLOGICAL EQUIVALENCE OF A BANACH SPACE WITH ITS UNIT CELL
}

\author{
BY VICTOR KLEE ${ }^{1}$
}

Communicated by Mahlon M. Day, January 3, 1961

Several years ago [8] we proved that Hilbert space is homeomorphic with both its unit sphere $\{x:\|x\|=1\}$ and its unit cell $\{x:\|x\| \leqq 1\}$. Later [9] we showed that in every infinite-dimensional normed linear space, the unit sphere is homeomorphic with a (closed) hyperplane and the unit cell with a closed halfspace. It seems probable that every infinite-dimensional normed linear space is homeomorphic with both its unit sphere and its unit cell, but the question is unsettled even for Banach spaces. Corson [4] has recently proved that every $\boldsymbol{\aleph}_{0}$-dimensional normed linear space is homeomorphic with its unit cell. In the present note, we establish the same result for a class of infinite-dimensional Banach spaces which is believed to include all such spaces. It is proved to include every infinite-dimensional Banach space which is reflexive, or admits an unconditional basis, or is a separable conjugate space, or is a space $C M$ of all bounded continuous real-valued functions on a metric space $M$.

We employ the following tools:

(1) If $E$ and $F$ are Banach spaces and $u$ is a continuous linear transformation of $E$ onto $F$, then there exist a constant $m \in] 0, \infty$ [ and continuous mapping $v$ of $F$ into $E$ such that $u v x=x, v r x=r v x$, and $\|v x\| \leqq m\|x\|$ for all $x \in F$ and $r \in R$ (the real number space). If $G$ is the kernel of $u$ and $h y=(u y, v u y-y) \in F \times G$ for each $y \in E$, then $h$ is a homeomorphism of $E$ onto $F \times G$. Let $\|(p, q)\|=\max (\|p\|,\|q\|)$ for all $(p, q) \in F \times G$, and let $\xi y=(\|y\| /\|h y\|) h y$ for all $y \in E$. Then $\xi$ is a homeomorphism of $E$ onto $F \times G$ which carries the unit cell of $E$ onto that of $F \times G$.

(2) If $S$ is a closed linear subspace of a Banach space $E$, then $E$ is homeomorphic with the product space $(E / S) \times S$ and the unit cell of $E$ is homeomorphic with the unit cell of this product space (with respect to any norm compatible with the product topology).

(3) In each infinite-dimensional normed linear space, the unit cell is homeomorphic with a closed halfspace.

(4) If $Q$ is an open halfspace in an infinite-dimensional normed linear space and $p$ is a point in the boundary of $Q$, then $Q \cup\{p\}$ is homeomorphic with $Q$.

${ }^{1}$ Research Fellow of the Alfred P. Sloan Foundation. 
(5) For each $\left.f \in L^{2}\right] 0, \infty[$ and $t \in[0,1[$, let the function $\left.f_{t} \in L^{2}\right] 0, \infty$ [ be defined as follows: $f_{t} x=t f(t x)$ for $\left.x \in\right] 0,1\left[; f_{t} x\right.$ $=f(x+t-1)$ for $x \in\left[1, \infty\left[\right.\right.$. Then with $\eta(f, t)=\left(f_{t}, t\right)$, the transformation $\eta$ is a homeomorphism of $\left.L^{2}\right] 0, \infty\left[\times\left[0,1\left[\right.\right.\right.$ onto $\left(L^{2}\right] 0, \infty[X] 0,1[)$ $\cup\left(L^{2}[1, \infty[\times\{0\})\right.$.

The existence of $v$ and $m$ as described in (1) follows from a theorem of Bartle and Graves [1, p. 404] (see also Michael [13]). It is easily verified that $h$ is a homeomorphism [10], and homogeneity of $h$ follows from that of $u$ and $v$. Thus the transformation $\xi$ is also homogeneous. To complete the proof of (1) it suffices to observe that $(1+m)^{-1}\|y\| \leqq\|h y\| \leqq(m\|u\|+1)\|y\|$ for all $y \in E$. Proposition (2) results from applying (1) to the canonical mapping $u$ of $E$ onto $E / S$.

The result (3) appears in [9]. For (5), see page 29 of [8]. A theorem much stronger than (4) is proved on pages 12-28 of [8]. When the space is nonreflexive or is an $\left(l^{p}\right)$ space, (4) is explicitly a corollary of (3.3) on page 27 of [8]. In the general case, it follows from the reasoning (though not explicitly from any statement) in [8]. Also, a proof of (4) is outlined in [11].

A normed linear space $J$ will be called compressible provided the space $J \times[0,1[$ is homeomorphic with the space $(J \times] 0,1[)$ $\cup(W \times\{0\})$ for some closed linear subspace $W$ of infinite deficiency in $J$. (We see by (5) that Hilbert space is compressible.) A space is $h$-compressible provided it is homeomorphic with some compressible normed linear space.

THEOREM. If a Banach space $B$ admits a continuous linear transformation onto a Banach space $E$ which contains an h-compressible closed linear proper subspace $S$, then $B$ is homeomorphic with the unit cell of $B$.

Proof. Let $G$ denote the kernel of the continuous linear transformation of $B$ onto $E$. By (1), $B$ is homeomorphic with the product space $P=E \times G$ and the unit cell of $B$ is homeomorphic with the unit cell $U$ of $P$. To establish the theorem, it suffices to show that $P$ is homeomorphic with $U$. Since $S$ is a closed linear proper subspace of $E$, the subspace $T=S \times\{0\}$ must be in a closed hyperplane $V$ in $P$. The unit cell $U$ of $P$ is homeomorphic with $V \times[0,1$ [ by (3), and $V$ is homeomorphic with $(V / T) \times T$ by (2), so $U$ is homeomorphic with $(V / T) \times(T \times[0,1[)$. Clearly $P$ itself is homeomorphic with $V \times] 0,1[$ and hence with $(V / T) \times(T \times] 0,1[)$, so to complete the proof it suffices to show that $T \times[0,1[$ is homeomorphic with $T \times] 0,1[$. Since $T$ is $h$-compressible, there exist a Banach space $J$ homeomorphic with $T$ and a subspace $W$ of infinite deficiency in $J$ such that 
$J \times[0,1$ [is homeomorphic with $(J \times] 0,1[) \cup(W \times\{0\})$. Let $u$ denote the canonical mapping of $J$ onto $J / W$ and then let $v$ and $h$ be as in (1) above. Then $h$ is a homeomorphism of $J$ onto $(J / W) \times W$, and since $h w=(\theta, v \theta-w)$ for all $w \in W$ (where $\theta$ is the neutral element of $J / W)$, it follows that $h W=\{\theta\} \times W$. Consequently the space $(J \times] 0,1[) \cup(W \times\{0\})$ is homeomorphic with

$$
(J / W) \times W \times] 0,1[\cup\{\theta\} \times W \times\{0\},
$$

which in turn is homeomorphic with

$$
W \times((J / W) \times] 0,1[\cup\{0\} \times\{0\}) .
$$

Since $J / W$ is infinite-dimensional, it follows by (4) that the set above is homeomorphic with

$$
W \times((J / W) \times] 0,1[),
$$

and hence with $J \times] 0,1[$. Reviewing the information now assembled, we see that $T \times[0,1[$ is homeomorphic with $T \times] 0,1[$, and hence that $U$ is homeomorphic with $P$. This completes the proof of the theorem.

Corollary. If an infinite-dimensional Banach space $B$ satisfies at least one of the following conditions, then $B$ is homeomorphic with its unit cell:

(a) $B$ is reflexive;

(b) $B$ is a linear subspace of a Banach space which admits an unconditional basis;

(c) $B$ is a norm-separable $w^{*}$-closed linear subspace of a conjugate space;

(d) $B$ is the space $C N$ of all bounded continuous real-valued functions on a normal space $N$ which contains a closed infinite metrizable subset.

Proof. In view of the theorem and the fact (by (5)) that Hilbert space is compressible, it suffices in each case to produce a continuous linear transformation of $B$ onto a Banach space $E$ which contains a closed linear proper subspace $S$ which is homeomorphic with Hilbert space. When $B$ is reflexive, let $E=B$ and let $S$ be an infinite-dimensional separable closed linear proper subspace of $E$. Then $S$ is reflexive and hence (by a theorem of Kadeč [7]) homeomorphic with Hilbert space.

If $B$ is a subspace of a space which admits an unconditional basis, a theorem of James [5] and Bessaga and Pełczyński [2] asserts that either $B$ is reflexive or some linear subspace of $B$ is linearly homeomorphic with the space $(l)$ or the space $\left(c_{0}\right)$. But the latter two spaces 
are known to be homeomorphic with Hilbert space (by results of Mazur [12] and Kadeč [6]) and the desired conclusion follows.

Now suppose $B$ is a separable conjugate space or, more generally, that $B$ is a norm-separable $w^{*}$-closed linear subspace of a conjugate Banach space $L^{*}$. Let $f \in B \sim\{0\}, x \in L$ with $f x=1$, and $S=\{g \in E: g x=0\}$. Then $S$ is a $w^{*}$-closed linear proper subspace of $B$, and must be homeomorphic with Hilbert space by a theorem in [10]. Consequently, $B$ is homeomorphic with its unit cell.

Finally, let $B$ and $N$ be as in (d). Then there is a countably infinite closed subset $Z$ of $N$ which consists of either a discrete set or a convergent sequence together with its limit point. For each $\phi \in C N$ let $u \phi=\phi \mid Z \in C Z$. Then $u$ is a continuous linear transformation of $C N$ onto $C Z$, and $C Z$ is equivalent to either the space $(m)$ or the space $\left(c_{0}\right)$. In either case, $C Z$ has the $h$-compressible space $\left(c_{0}\right)$ as a closed linear proper subspace, and the desired conclusion follows upon applying the theorem.

Note that the topological equivalence of every infinite-dimensional Banach space with its unit cell would be implied by the generally expected affirmative answer to the following question: Are all infinite-dimensional separable Banach spaces homeomorphic? Recent results on this problem have been obtained by Bessaga and Pełczyński [3].

At least for reflexive spaces, the corollary above can be significantly improved. The method is that of $[8, \mathrm{pp} .30-31]$ in conjunction with the above techniques and the result is as follows:

Theorem. Suppose $E$ is an infinite-dimensional reflexive Banach space and $C$ is a closed convex subset of $E$ which has nonempty interior. Then $C$ is homeomorphic with $E$ and the boundary of $C$ is homeomorphic with $E$ or with $E \times S^{n}$ for some finite $n$ and $n$-sphere $S^{n}$.

The following problems seem worthy of mention: Are all infinitedimensional separable Banach spaces $h$-compressible? (An affirmative answer implies that every infinite-dimensional Banach space is homeomorphic with its unit cell.) Are all infinite-dimensional Banach spaces compressible? Are $\boldsymbol{\aleph}_{0}$-dimensional normed linear spaces compressible? Note that for Hilbert space, the compressibility was achieved by means of a continuous family of affine homeomorphisms. How generally is this possible?

\section{REFERENCES}

1. R. G. Bartle and L. M. Graves, Mappings between function spaces, Trans. Amer. Math. Soc. vol. 72 (1952) pp. 400-413. 
2. C. Bessaga and A. Pelczytski, A generalization of results of $R$. C. James, Studia Math. vol. 17 (1958) pp. 165-174.

3. - Some remarks on homeomorphism of Banach spaces, to appear.

4. H. H. Corson and Victor Klee, Topological classification of convex bodies, to appear.

5. R. C. James, Bases and reflexivity of Banach spaces, Ann. of Math. vol. 52 (1950) pp. 518-527.

6. M. I. Kadex, On homeomorphism of certain Banach spaces, Dokl. Akad. Nauk SSSR vol. 92 (1953) pp. 465-468 (Russian).

7. - On weak and norm convergence, Dokl. Akad. Nauk SSSR vol. 122 (1958) pp. 13-16 (Russian).

8. Victor Klee, Convex bodies and periodic homeomorphisms in Hilbert space, Trans. Amer. Math. Soc. vol. 74 (1953) pp. 10-43.

9. - A note on topological properties of normed-linear spaces, Proc. Amer. Math. Soc. vol. 7 (1956) pp. 673-674.

10. - Mappings into normed linear spaces, Fund. Math. vol. 49 (1960) pp. 25-34.

11. - Topological structure of infinite-dimensional linear spaces, to appear.

12. S. Mazur, Une remarque sur l'homeomorphic des champs fonctionnels, Studia Math. vol. 1 (1929) pp. 83-85. 382.

13. Ernest Michael, Continuous selections. I, Ann. of Math. vol. 63 (1956) pp. 361-

\section{UNIVERSITY OF WASHINGTON}

